\title{
A ADMINISTRAÇÃO DO LUCRO CONTÁBIL E OS CRITÉRIOS PARA DETERMINAÇÃO DA EFICÁCIA DO HEDGE ACCOUNTING: UTILIZAÇÃO DA CORRELAÇÃO SIMPLES DENTRO DO ARCABOUÇO DO SFAS $\mathrm{N}^{0} 133^{1}$
}

\author{
Alexsandro Broedel Lopes \\ Professor Doutor do Departamento de Contabilidade e Atuária da FEA-USP \\ Nelson Seixas dos Santos \\ Doutorando em Economia pela FEA-USP
}

O hedge accounting constitui inovação relevante introduzida pelo SFAS 133. Dentro desse critério, operações designadas como hedge deverão ter seus resultados diferidos para o momento no qual os itens sendo protegidos forem reconhecidos. Problema central nessa contabilização é a determinação do quê constitui uma operação de hedge. Os órgãos reguladores analisados neste trabalho apresentam critérios ad hoc para a determinação da eficácia das operações de hedge. Esses critérios não possuem propriedades estatísticas de previsão do comportamento dos ativos no futuro, condição necessária para o sucesso do hedge. Esse tipo de qualificação de hedge introduz enorme subjetividade no processo de reconhecimento contábil, fornecendo ampla margem para manipulação dos resultados. Este trabalho analisa e critica a opção adotada pelo FASB e outros órgãos reguladores e oferece sugestões.

Palavras-chave: administração de resultados; eficácia do hedge; correlação; SFAS 133.
The hedge accounting mechanism is a recent innovation introduced by SFAS 133. According to this method, transactions designated as hedge should have their results deferred to a future period when the hedged items are recognised. Hedge characterisation is a central problem in this subject. Regulatory bodies studied in this work use ad hoc criteria to determine hedge effectiveness. Those criteria are not statistically significant in terms of forecasting the asset's behaviour, a necessary condition for a successful hedge. This sort of hedge characterisation introduces more subjectivity into accounting recognition, allowing for manipulation. This work criticises the option adopted by FASB and other regulatory bodies.

Keywords: earnings management; hedge effectiveness; correlation; SFAS 133. 


\section{INTRODUÇÃO}

O mercado financeiro internacional apresentou nas últimas décadas um aumento significativo na volatilidade dos principais produtos negociados, resultando em um crescimento considerável no risco de mercado das operações realizadas ${ }^{2}$ (JORION, 1997: 28). Esse aumento de volatilidade deve-se a um grande número de fatores institucionais e econômicos que levaram as instituições financeiras internacionais a se defrontar com um ambiente muito mais instável e com possibilidades de perda bastante exacerbadas.

Esse novo panorama de risco crescente nas operações financeiras levou o mercado ao desenvolvimento de novos instrumentos financeiros - os derivativos - para amenizar as conseqüências inerentes a operações realizadas dentro de um ambiente adverso. O papel desses instrumentos como meios de gestão e controle de riscos é fundamental na medida em que permitem a transferência de riscos entre agentes diversos, possibilitam a garantia de fixação de preços e taxas futuras e ainda permitem a criação de instrumentos sintéticos por intermédio da utilização de vários outros instrumentos com diferentes perfis de fluxos de caixa.

No entanto, a própria operação com os instrumentos financeiros derivativos apresenta enormes riscos (PERRY, 1996:1). Estes riscos advêm basicamente da capacidade de alavancagem inerente aos derivativos, a qual possibilita perdas muitas vezes superiores ao volume de recursos investidos. Aliado aos problemas relativos à alavancagem nas operações com derivativos, existe o risco operacional derivado do desconhecimento de muitos profissionais envolvidos com estas atividades em relação à complexidade inerente com tais operações. Podemos observar em JORION (1997: 28) que as perdas sofridas pelas instituições financeiras em operações com derivativos têm aumentado ao longo dos anos na década de 90 .

Dessa forma, pode-se perceber que a utilização dos derivativos, apesar de possuir um grande potencial para redução dos riscos envolvidos nas atividades financeiras, também pode causar prejuízos significativos às instituições envolvidas. Como conseqüência desta dualidade, esses instrumentos passaram a ser classificados em duas categorias básicas (FASB, 1998:2):
- Trading instruments: são os contratos realizados com o objetivo de auferir lucros com as flutuações de preços e taxas no mercado; esses instrumentos aumentam a alavancagem da instituição, uma vez que juntamente com este aumento no potencial de geração de lucros há um conseqüente aumento, não necessariamente proporcional, nos riscos de prejuízos com as operações realizadas;

- Hedging Instruments: são aqueles que visam a proteger a instituição de prejuízos advindos das flutuações de preços e taxas no mercado sobre os seus ativos ou passivos. Esses instrumentos têm como objetivo compensar as variações ocorridas nos ativos ou passivos, anulando as variações desfavoráveis ocorridas.

Essa classificação procura segregar as operações com derivativos que têm como objetivo a geração de resultados daquelas que têm como objetivo a redução dos prejuízos. Com essa segregação é possível identificar qual o perfil das operações realizadas por uma instituição e conseqüentemente analisar qual o nível de exposição ao risco que ela está assumindo em suas atividades.

No entanto, essa classificação é dependente de um critério de determinação de quais operações realmente se enquadram como hedge. Em operações com perfil de proteção tradicional, como utilizar opções de venda no mesmo título para proteger uma carteira à vista de ações em um fundo de investimentos, não existem grandes problemas. Os problemas surgem à medida em que são feitas operações para proteção em moedas, commodities ou títulos diferentes, como café brasileiro e café colombiano, por exemplo.

Por tudo isso, objetiva-se, neste trabalho, analisar a estrutura conceitual da utilização das operações financeiras de hedge e seus impactos para a contabilidade, bem como sugerir algumas alternativas de mensuração.

\section{JUSTIFICATIVAS E ESCOPO DO TRABALHO}

Como conseqüência das alterações mencionadas no mercado financeiro, o estabelecimento de um critério claro para determinação de quais operações 
realmente se encaixam na categoria de hedge tornouse uma prioridade para órgãos reguladores do mercado financeiro e normatizadores da Contabilidade. Ademais, tais órgãos mostram-se interessados em determinar a extensão em que a proteção é eficaz, bem como a evidenciação dessas informações pelas instituições.

Dentro deste cenário, o Financial Accounting Standards Board-FASB emitiu em junho de 1998 o pronunciamento № 133, Accounting for Derivative Instruments and Hedging Activities, no qual estabeleceu critérios diferentes para a contabilização de operações classificadas como trading ou hedge activities. Por conseguinte, a partir de junho de 1999, tornou-se obrigatório o estabelecimento de critérios para a classificação das operações de hedge, para todas as empresas que precisam apresentar suas demonstrações dentro dos princípios de contabilidade geralmente aceitos nos EUA (United States - General Accepted Accounting Principles, US-GAAP), sejam elas americanas ou estrangeiras, que buscam o mercado americano.

Além desta justificativa de caráter obrigatório, do ponto de vista gerencial, é extremamente importante para a administração da organização o conhecimento de quais são as operações que se encaixam como operações de hedge. Portanto, a determinação de um critério realmente eficaz auxilia bastante os gestores na eliminação de ineficiências em operações de hedge que aparentemente são coerentes, mas na realidade não se observa o esperado.

Este trabalho procura realizar uma análise teórica abrangente a respeito dos critérios de hedge e conseqüentemente do seu tratamento pela contabilidade (hedge accounting). A atenção estará sempre voltada para o risco de mercado das operações, sem considerar riscos operacionais, de crédito etc. Assim, somente serão abordadas as operações com derivativos tradicionais e realizadas no mercado brasileiro, não sendo contempladas as opções exóticas, os derivativos de crédito e o clima, entre outros.

\section{HEDGE ACCOUNTING: ESTRUTURA CONCEITUAL}

Recentemente, tem-se desenvolvido uma contabilidade especial para operações de derivativos designadas para proteger riscos de mercado de ativos e passivos (hedge accounting). De fato, isso resulta do princípio da confrontação da receita e da despesa (matching principle) que reza que as receitas e despesas relativas a uma operação sejam reconhecidas no mesmo período. Dessa forma, em muitas operações de hedge, os resultados da operação de derivativos realizada para proteger um determinado ativo ou passivo não devem ser contabilizados no período de sua ocorrência e sim quando o item sendo "hedgeado" tem a sua variação.

Outrossim, uma operação de futuros realizada para proteger uma dívida de longo prazo de uma variação cambial teria o resultado de seus ajustes diários diferido até o momento em que houvesse o impacto na dívida advindo da variação cambial respectiva. Só então haveria uma reclassificação do resultado da operação de futuros para a conta de resultado. Com esta metodologia, o resultado da operação de derivativos teria impacto no resultado no mesmo período do item sendo protegido e, caso o hedge seja perfeitamente eficaz, este impacto seria nulo.

A demanda por uma contabilidade especial para operações de hedge fortalece-se por algumas anomalias no processo de reconhecimento contábil (FASB, 1998:156). Tais anomalias advêm de diferenças no processo de reconhecimento e mensuração de itens sendo utilizados para hedge (hedging instruments) e itens sendo protegidos (hedged instruments). Estas anomalias relativas ao reconhecimento surgem porque alguns ativos e passivos são reconhecidos nas demonstrações enquanto outros, como compromissos firmes e transações esperadas, não o são. Anomalias de mensuração aparecem na medida em que os padrões contábeis indicam métodos de mensuração diferentes para diferentes categorias de ativos e passivos; alguns ativos e passivos são mensurados pelo custo histórico, enquanto outros - são pelo valor corrente entre outras metodologias. Assim, o hedge accounting surge como uma forma de compensar as diferenças de reconhecimento e mensuração entre os elementos envolvidos em uma operação de hedge.

A utilização de uma metodologia de hedge accounting deriva diretamente da estrutura 
conceitual básica da contabilidade. Receitas e despesas devem ser confrontadas. Uma operação realizada para proteger uma dívida em moeda estrangeira, por exemplo, deve ter seu resultado reconhecido no momento do reconhecimento do resultado da obrigação. Isso decorre do fato de o instrumento financeiro utilizado para proteger a dívida ter sua natureza intimamente ligada com essa obrigação. Esse instrumento financeiro não existiria sozinho no contexto do hedge mencionado. Dessa forma, seu reconhecimento não deve ser feito de forma independente do item sendo hedgeado.

A utilização desta contabilidade especial também possui ramificações na medida em que podemos considerar várias categorias de hedge para riscos de mercado. Podemos pensar em estruturas de proteção para fluxos de caixa ou para o fair value das operações, dependendo do motivo que levou à realização da operação em questão. A estrutura conceitual apresentada é independente do item sendo protegido. Desde que a proteção possa ser comprovada objetivamente, o mesmo arcabouço se aplica.

Para o usuário externo da informação contábil, a utilização do hedge accounting possui uma vantagem natural advinda da segregação entre as operações de proteção e as operações destinadas à especulação. Com esta separação, o analista de investimentos, por exemplo, poderá identificar de imediato qual o montante de operações que estão sendo realizadas pela organização com o objetivo de proteger as suas posições e qual o resultado dessas operações.

Como podemos observar em PERRY (1996:32), o desenvolvimento da estrutura conceitual para realização do hedge accounting tem sido realizado na medida em que os problemas vão surgindo, constituindo-se, assim, um corpo teórico conceitualmente desorganizado e falho. Dentro desta estrutura, o aparecimento do SFAS № 133 do FASB vem preencher uma certa lacuna neste cenário, na tentativa de fornecer um arcabouço definitivo e homogeneizado para todas as operações com derivativos (WILSON, 1998:25). Assim, percebe-se que os estudos no sentido de integralizar um corpo de conhecimentos definitivos para este modelo de contabilização são ex- tremamente importantes, bem como estudos visando ao desenvolvimento de critérios definitivos para verificação da eficácia do hedge.

\section{MÉTODOS PARA DETERMINAÇÃO DA EFICÁCIA DO HEDGE ACCOUNTING}

Dentro dos estudos que visam à criação de um modelo conceitual definitivo para as operações de hedge accounting, uma questão ocupa lugar central nesta problemática: qual o melhor método para a determinação da eficácia do hedge?

Esta questão assume um contorno muito importante, pois, na medida em que se define qual o critério para determinação da eficácia de uma operação de hedge, também se definem quais operações são de hedge e quais são de trading. Isso ocorre, porquanto há necessidade de se estabelecer um limite de eficácia abaixo do qual a operação será considerada uma operação com finalidades especulativas.

Este critério é necessário, pois não se pode considerar hedge uma operação que se mostra consistentemente incapaz de cobrir os prejuízos causados pela operação que pretende hedgear. Em suma, temse em questão qual o limite de ineficiência admitido, a metodologia de cálculo desse limite e a agregação das operações que devem ser consideradas.

Existem várias considerações que devem ser realizadas na determinação de uma relação de hedge:

- escopo e abrangência da relação: deve ser determinado se a relação se refere a uma única operação, um conjunto de operações ou à organização como um todo;

- durabilidade da relação: a qual período se refere a relação de hedge considerada? Este período deve ser determinado para que se possa estabelecer um mecanismo de cálculo da relação de hedge estabelecida;

- término da relação: critérios específicos para determinação do fim da relação de hedge analisada;

- freqüência: deve ser estabelecido qual o período considerado para verificação da relação inicialmente estabelecida;

- metodologia de cálculo: a metodologia estabelecida deve ser consistente ao longo de toda a duração do contrato.

Neste trabalho, analisa-se principalmente o problema da determinação de uma metodologia para determinação da eficácia do hedge considerado. Torna-se ne- 
cessária a determinação de um método quantitativo objetivo e claro para a verificação do grau de eficácia do hedge. Infelizmente, a bibliografia contábil tem se mostrado bastante pobre no que concerne ao fornecimento de mecanismo de verificação da eficácia das operações de hedge como podemos ver em STRAUSS (1998:45). A maioria dos textos que tratam desse assunto não fornecem uma metodologia definitiva, fornecendo somente uma abordagem superficial e indicando que as operações de hedge devem cobrir todas as variações nos elementos sendo "hedgeados" (PERRY, 1996:215).

Por isso, a solução de tal problema carece de ser procurada fora dos domínios contábeis. Nesse sentido, torna-se assaz importante colocar o problema em termos tão genéricos quanto possível, isto é, descrevê-lo em termos intuitivos. Em consonância com o exposto, definamos hedge como segue:

\section{Definição 1: Hedge}

Diz-se que uma operação H é hedge de uma outra operação $\mathrm{T}$, se o retorno da carteira de ativos transacionada em $\mathrm{H}$ tiver sempre o sinal contrário ao retorno da carteira de ativos transacionada na operação $\mathrm{T}$.

Sendo assim, para que uma operação $\mathrm{H}$ se constitua em hedge de uma operação $\mathrm{T}$, basta que o retorno da carteira de ativos transacionada em $\mathrm{H}$ guarde uma relação linear negativa com o retorno da carteira de ativos transacionada em T.

Estatisticamente, a forma mais empregada para medição do grau de relacionamento linear entre duas variáveis é dada pela correlação. Tal medida fornece como resultado um valor entre -1 e 1; uma correlação igual a1 indica que, se uma das variáveis crescer, por exemplo, $10 \%$, a outra cairá exatamente $10 \%$. Pelo contrário, correlação igual a 1 indica que, se uma das variáveis crescer, por exemplo, $10 \%$, a outra crescerá exatamente $10 \%$. Correlação nula indica que o crescimento de uma das variáveis não afeta a outra.

Em virtude disso, a partir do SFAS № 80 Accounting for Futures Contracts, a maior parte dos órgãos reguladores, em especial o FASB, passa a adotar a correlação estatística como critério de determinação da eficácia do hedge. A título de completeza, apresentaremos formalmente a:

\section{Definição 2: Correlação:}

$$
\rho \equiv \sigma_{x y} / \sigma_{x} \sigma_{y}
$$

onde:

$\rho$ : correlação

$\sigma_{\mathrm{xy}}$ : covariância da variável $\mathrm{x}$ com a variável y

$\sigma_{x}$ : desvio-padrão da variável $x$

$\sigma_{\mathrm{y}}$ : desvio-padrão da variável y

Apesar de sua facilidade de cálculo, o emprego da correlação estatística como método de determinação da eficácia de operações de hedge sofre de uma seríssima dificuldade ${ }^{3}$. De fato, a existência de elevada correlação entre duas variáveis em um determinado período de tempo não é garantia de que esta correlação continuará a existir no futuro. Portanto, se classificarmos uma operação com base na correlação, poder-se-á com grande probabilidade considerar como hedge operações que, em futuro próximo, mostrar-se-ão ineficazes na proteção de riscos e que podem até, pelo contrário, exacerbar ainda mais o risco das operações.

Por isso, seria necessário proceder continuamente a testes para verificar se a relação entre os retornos mencionada se mantém ao longo de todo o período das operações de hedging e hedgeada, tornando o trabalho de controle das operações excessivamente difícil e oneroso. Com efeito, o problema de determinação da existência de um relacionamento de longo prazo entre duas séries de tempo de variáveis econômicas não é trivial e, por ser muito peculiar à análise econômica, só obteve um tratamento aprofundado nos domínios da Econometria. ${ }^{4}$

Do ponto de vista econométrico, a primeira coisa a ser feita é coletar uma amostra relativamente gran$\mathrm{de}^{5}$ de cada série de retorno e observar cada uma delas como proveniente de um processo estocástico,

\footnotetext{
${ }^{3}$ O FASB também oferece a possibilidade do uso simples da cobertura percentual dentro dos limites de 80 a $125 \%$ da variação do item sendo protegido. Assim, um derivativo deve cobrir entre 80 e $125 \%$ da variação do ativo ou passivo sendo protegido para que possa ser designado como hedge. As críticas apresentadas à correlação são diretamente aplicáveis à cobertura percentual. O Banco Central do Brasil, por intermédio da Circular $\mathrm{N}^{\circ} 3082$, utiliza-se da cobertura percentual como parte dos critérios para a determinação da eficácia das operações de hedge.

${ }^{4}$ Econometria é o ramo da teoria econômica que se ocupa em determinar quantitativamente as relações empíricas entre as variáveis econômicas, estimando seus valores e testando hipóteses a seu respeito.

${ }^{5} \mathrm{Na}$ prática, uma amostra com 50 observações de uma variável pode ser considerada relativamente grande.
} 
isto é, uma seqüência temporal de variáveis aleatórias. A partir de então teremos de empregar a metodologia proposta em BOX-JENKINS (1976) e, se for o caso, proceder aos testes de raiz unitária propostos em DICKEY \& FULLER (1979) e PHILLIPS \& PERRON (1988) e, até mesmo, recorrer à análise de cointegração proposta em GRANGER (1983) e ENGLE \& GRANGER (1987). Essas técnicas adicionam considerável consistência ao uso da correlação estatística.

Outro caminho seria adotar a metodologia proposta em HARVEY (1989), mas, de qualquer modo, não é possível furtar-se ao uso de métodos econométricos mais poderosos, se realmente se pretende estabelecer um critério consistente para determinação da eficácia do hedge tal como o definimos. ${ }^{6}$

Sucintamente, pode-se afirmar que a correlação não é a forma mais consistente de se proceder à análise de séries históricas e determinação de relações futuras. De fato, apenas com o emprego de técnicas econométricas mais sofisticadas e robustas poder-se-á estabelecer com firmeza tais relações. Outro aspecto que deve ser enfatizado é a relação entre a significância estatística e a relevância econômica. Atenção especial deve ser dada a relações espúrias e estatísticas mal calculadas que podem levar a tomadas de decisão que contrariam o sentido econômico das operações.

\section{HEDGE ACCOUNTING E DERIVATIVOS: O SFAS № 133}

O FASB, por intermédio do SFAS № 133, introduziu mudanças na contabilização das operações com derivativos classificadas como hedge da seguinte forma:

- mensuração dos Derivativos pelo Fair Value: O FASB esclarece que o fair value é a melhor medida de mensuração de instrumentos financeiros e a única medida possível para os derivativos. Dessa forma, todos os derivativos devem ser reconhecidos nos balanços de acordo com o fair value dos direitos e das obrigações conferidos aos participantes. A melhor medida de valor justo é considerada o valor de mercado dos produtos que forem negociados em bolsas de derivativos e similares. Para os derivativos que não possuírem esta ca- racterística de negociação podem ser usados vários modelos para a obtenção desses valores: valor presente de fluxos futuros de caixa, precificação de opções (Black\&Scholes por exemplo), modelo binomial e vários outros; analogias com outros instrumentos também podem ser consideradas. No entanto, é fundamental que os modelos adotados estejam bem explicitados nas notas explicativas e que a sua aplicação seja consistente ao longo dos períodos. Pode-se considerar que este é um passo importante da Contabilidade, uma vez que está se abrindo a hipótese da contabilização de um valor estimado que não é necessariamente objetivo; e se os modelos de precificação fornecessem medidas seguras de preço destes produtos, não haveria mercado para tal negociação.

- classificação dos Produtos de acordo com a finalidade para a qual eles foram adquiridos:O Board decidiu adotar as seguintes classificações para as operações de derivativos de acordo com a finalidade para a qual a entidade os adquiriu:

1. Hedge de uma exposição a mudanças no fair value de um ativo ou passivo já reconhecido ou de um compromisso firme ainda não reconhecido (fair value hedge).

2. Hedge de uma exposição a fluxos de caixa variáveis de uma transação futura projetada (cash flow hedging).

3. Hedge de uma exposição a uma moeda estrangeira de um investimento em uma operação internacional, um compromisso ainda não reconhecido, um título destinado à operação de trading da instituição ou uma transação esperada denominada em moeda estrangeira.

4. Instrumentos não designados como instrumentos de hedge. Estes instrumentos são aqueles que a instituição adquire com o propósito de obter resultados positivos com as flutuações de preços e taxas.

Esta classificação possui uma importância enorme, pois orienta todo o tratamento contábil subseqüente destes produtos.

- a contabilização dos ganhos e perdas com esses produtos (variações de fair value) depende da intenção no uso do instrumento e de sua respectiva classificação. Para cada uma das classificações apresentadas acima, o SFAS 133 determina uma forma específica de reconhecimento dos ganhos e perdas para cada derivativo

${ }^{6}$ CAMPBELL et al. (1997) apresentam um excelente texto de análise econométrica do mercado financeiro. 
considerado. Dessa forma, temos a seguinte orientação:

1. Fair Value Hedge: os ganhos ou perdas são reconhecidos no resultado no período de variação juntamente com o resultado do item sendo protegido, atribuído ao risco sendo considerado. Dentro desta metodologia, somente será levado ao resultado a parte não eficaz no processo de anulação das perdas ou ganhos no item protegido.

2. Cash Flow Hedge: as variações são mostradas inicialmente como parte de outros ganhos, fora do resultado, e subseqüentemente reclassificadas para o resultado quando a transação futura vier a ocorrer.

3. Hedge de uma Exposição em Moeda Estrangeira de um Investimento em uma Operação Internacional: os ganhos ou perdas são classificados em outros ganhos, fora do resultado, como uma parte do ajuste acumulado da conversão. O modelo de contabilização de Fair Value Hedge descrito acima aplica-se a um derivativo designado como hedge de uma exposição em moeda estrangeira de um compromisso firme não reconhecido ou de um título utilizado para a operação normal da empresa. Da mesma forma, a contabilidade de um Cash Flow Hedge descrito acima aplica-se a todos os derivativos designados como hedge de uma transação projetada em moeda estrangeira.

4. Instrumentos não Classificados como Hedge: neste caso, os ganhos ou perdas são reconhecidos no resultado imediatamente.

Apesar de o SFAS № 133 não obrigar as instituições a utilizarem a correlação estatística como forma de determinação do hedge, ele a recomenda (FASB,1998:177) de forma semelhante ao SFAS № 80 , onde é recomendado que uma correlação de módulo acima de 0,80 possa classificar uma operação como hedge accounting (PERRY,1996:137). Assim, percebese que, com a obrigatoriedade do referido pronunciamento a partir de junho de 1999, a questão da determinação da correlação entre variáveis financeiras será de importância fundamental para todas as organizações que se utilizarem do pronunciamento do FASB.

\section{UTILIZAÇÃO DA CORRELAÇÃO PARA DETERMINAÇÃO DA EFICÁCIA DO HEDGE NO SFAS № 133}

Como foi observado no texto acima, a determinação da relação de hedge dentro da estrutura do SFAS № 133 acarreta uma série de conseqüências para a contabilização dos produtos financeiros negociados pela organização. A classificação de uma operação como hedge possibilita o diferimento do resultado do derivativo para posterior confrontação com o item sendo hedgeado. Dessa forma, se uma operação de trading é incorretamente classificada como hedge, teremos o diferimento do resultado de forma incorreta, impactando o lucro ou prejuízo da organização.

Assim, como foi verificado, a utilização da correlação estatística simples produz resultados enganosos que levam a anomalias de mensuração, baseadas na metodologia sugerida pelo Board do FASB em seu último pronunciamento. É fundamental ressaltar que esses problemas não podem ser justificados pela complexidade operacional dos procedimentos econométricos aqui recomendados, uma vez que o pronunciamento № 133 de uma forma geral é bastante complexo e de difícil aplicação prática (STRAUSS, 1998: 68). Sendo assim, a introdução desta nova metodologia não aumentaria, de forma desproporcional ao benefício encontrado, a complexidade do referido pronunciamento.

O IASB (International Accounting Standards Board) também adota a mesma metodologia sugerida pelo FASB para a determinação da eficácia do hedge em seu pronunciamento № 39 , replicando os mesmos problemas citados anteriormente neste trabalho.

\section{O HEDGE ACCOUNTING E A 'ADMINISTRAÇÃO' DO RESULTADO CONTÁBIL}

Casos recentes, como a falência da Enron, estão gerando dúvidas sobre a confiabilidade da Contabilidade. Várias citações na imprensa especializada (The Economist, 2002, por exemplo) têm demonstrado preocupação com a ampla margem de manipulação que os contadores têm dentro dos padrões contábeis geralmente aceitos. Essas críticas são importantes por uma razão muito simples: perda de confiabilidade. Como intermediários informacionais, contadores e auditores dependem muito da credibilidade da informação 
fornecida. A perda da credibilidade inviabiliza os serviços prestados por auditores e contadores.

Dentro desse entendimento, os critérios de hedge accounting introduzidos pelo SFAS $133 \mathrm{e}$ abraçados pelo Banco Central brasileiro dentro da Circular № 3.082 introduzem grande subjetividade ao processo de reconhecimento e mensuração contábil. Dessa forma, o contexto não poderia ser pior para a introdução de um normativo com essas características. $\mathrm{O}$ alto grau de subjetividade e mesmo de incorreção técnica da utilização da correlação simples dá grande margem à manipulação dos resultados.

Naturalmente, as observações acima levam em consideração o momento institucional da contabilidade internacional. A quebra de assimetria informacional fica nitidamente prejudicada com o aumento da subjetividade possível dentro dos pronunciamentos contábeis. Dessa forma, os critérios de determinação da eficácia do hedge não contribuem para o aumento da relevância da informação contábil, nem para a maior segurança do sistema de fornecimento de informações financeiras para o mercado.

Obviamente, os aspectos levantados acima demandam investigações empíricas adicionais dentro do paradigma positivo. Atualmente, esse tipo de investigação é dificultado pela novidade dos pronunciamentos que tratam da figura do hedge accounting. Evidências empíricas adicionais podem ser obtidas com o surgimento de bases de dados confiáveis. Essas evidências podem corroborar, ou não, as hipóteses levantadas neste trabalho. Adicionalmente, questões institucionais podem influenciar nos resultados empíricos. Essas questões referem-se às características de governança dos mercados, bem como aos setores de atividade das empresas investigadas.

É importante ressaltar que existem outras alternativas para o entendimento do processo regulatório (captura regulatória, por exemplo) que poderiam gerar conclusões divergentes sobre o papel do hedge accounting na economia, uma vez que esse é um assunto extremamente regulamentado. Neste trabalho, essas e outras abordagens não são consideradas. Este trabalho está fundamentado nos aspectos básicos da teoria econômica e não em outras análises.

\section{CONCLUSÕES E SUGESTÕES PARA NOVAS PESQUISAS}

O estudo das operações financeiras com relação aos critérios de determinação da eficácia das estratégias de hedge se torna extremamente importante na medida em que o risco inerente ao mercado financeiro internacional aumenta por diversas razões de natureza estrutural. Na medida em que as instituições procuram novas alternativas para protegerem-se desses riscos, um estudo objetivo, visando a determinar a relação efetiva entre as variáveis básicas do mercado, tornase indispensável.

A Contabilidade responde à elevada demanda por operações de proteção com a utilização de uma metodologia especial para registro e evidenciação: o hedge accounting. Nesta metodologia, o resultado das operações de hedge é diferido para ser registrado juntamente com as operações sendo "hedgeadas". Dessa forma, é respeitado o princípio da confrontação entre a receita e a despesa, com o impacto no resultado sendo estabelecido somente pela parte ineficaz do hedge; assim, em operações de hedge perfeitas não há impacto no resultado.

O SFAS № 133 veio preencher uma certa lacuna de pronunciamentos objetivos para determinação da utilização dessas estratégias de hedge para operações com derivativos. Este pronunciamento estabelece uma contabilização especifica, de acordo com a categoria em que se encaixa a operação: fair value, cash flow ou foreign currency hedge. Neste pronunciamento, o Board do FASB estabelece a necessidade de se estabelecer critérios objetivos para determinação da eficácia do hedge realizado pelas corporações. O FASB não obriga, mas indica a utilização da correlação estatística para determinação da eficácia do hedge realizada e assim ser procedida a contabilização.

Cabe ressaltar, no entanto, que a correlação estatística (ou o método da cobertura percentual) não é um bom indicador de comportamento futuro de séries de tempo. Na realidade, não há evidências estatísticas de que duas variáveis altamente correlacionadas no presente venham a possuir qualquer tipo de correlação no futuro. Para determinação deste tipo de correlação para perí- 
odos futuros, outras técnicas mais sofisticadas como a cointegração deveriam ser utilizadas para que os resultados obtidos sejam mais robustos.

Pesquisas futuras no sentido de encontrar técnicas econométricas mais apropriadas para determinação de correlação futura se fazem necessárias na medida em que cresce a importância dos estudos de proteção quanto ao risco financeiro em face de um mercado mais volátil, como as recentes crises no mercado cambial brasileiro podem atestar. Estudos desta natureza se mostram importantes na medida em que reduzem a incerteza relativa à determinação deste tipo de relação e melhoram o grau de conhecimento dos agentes econômicos sobre o assunto, fornecendo insumos interessantes para as estratégias adotadas.
Outra linha de pesquisa que promete gerar frutos no futuro refere-se ao estudo analítico da evidenciação das operações de hedge nas decisões de investimento dos agentes. Isso se refere-se ao impacto da evidenciação contábil na economia 'real'. Questões como essa começaram a ser investigadas por autores como SAPRA (2002), que tentam demonstrar que o nível informacional gerado pelo disclosure pode impactar o processo de formação de preços e as ações tomadas pelos administradores na economia. Esse tipo de pesquisa analítica tem o potencial para gerar insights para o teste empírico do papel relativo do hedge accounting no nível geral de informação do mercado.

\section{REFERÊNCIAS BIBLIOGRÁFICAS}

BOX, G.E.P and JENKINS, G. M. Time Series Analysis: Forescasting and Control. San Francisco: Holden-Day, 1976.

CAMPBELL, J.; LO, A.; MACKINLAY, C. The Econometrics of Financial Markets. Princeton, $\mathrm{NJ}$ : Princeton University Press, 1997.

DICKEY, D.A. \& FULLER, W.A. Distribution of the estimators for autoregressive time series with a unit root. Journal of American Statistical Association, 74(366), p.427-431, 1979.

ECONOMIST, The. When the numbers don't add up. February $9-15^{\text {th }} 2002$.

ENGLE, R.F. \& GRANGER, C.W. J. Cointegration and Error-Correction: Representation, Estimation and Testing. Econometrica, 55, p. 251-276, 1987.

FASB Exposure Draft, Accounting for Derivatives and Similar Financial Instruments and for Hedging Activities, 1996.

FASB. Statement of Financial Accounting Standards № 105. Disclosure of Information about Financial Instruments with off-balance-sheet risk and Financial Instruments with Concentrations of Credit Risk, 1990.
FASB. Statement of Financial Accounting Standards № 107. Disclosure about Fair Value of Financial Instruments, 1991.

FASB. Statement of Financial Accounting Standards № 115. Accounting for Debt and Equity Securities, 1993.

FASB. Statement of Financial Accounting Standards № 133. Accounting for Derivative Instruments and Hedging Activities, 133.

FASB. Statement of Financial Accounting Standards № 52. Foreign Currency Translation, 1981.

FASB. Statement of Financial Accounting Standards № 80, Accounting for Futures Contracts, 1984.

FASB. Statement of Financial Accounting Standards № 119, Disclosure about Derivative Financial Instruments and Fair Value of Financial Instruments, 1994.

GRANGER, C.W.J. Co-integrated Variables and ErrorCorrecting Models. Unpublished, University of California at San Diego Discussion Paper 83-13, 1983. 
HARVEY, C. Forecasting Structural Time Series Models and the Kalman Filter. Cambridge: Cambridge University Press, 1989.

JORION, Philip. Value at Risk. Nova York: Wiley, 1997.

LOPES, Alexsandro Broedel; LIMA, Iran Siqueira. Análise Comparativa dos Procedimentos Contábeis Aplicados a Operações Financeiras com Derivativos: um Estudo Brasil vs. Estados Unidos. Resenha da Bolsa de Mercadorias e Futuros. № 121 , janeiro de 1998. São Paulo.

LOPES, Alexsandro Broedel; LISBOA, Lázaro Plácido. Disclosure de Operações com Derivativos: um Estudo Comparativo Brasil vs. EUA. Resenha da Bolsa de Mercadorias e Futuros. № 124, agosto de 1998. São Paulo.

NAKAMURA, Angela Mie. Contribuição ao Estudo dos Procedimentos e Evidenciação Contábeis Aplicáveis a Operações com Derivativos, Voltados a Instituições Financeiras. 1996. Tese de Doutorado Apresentada ao Departamento de Ciências Contábeis e Atuariais da FEA/USP.

PERRY, Raymond E. Accounting for Derivatives. Chicago: Richard D. Irwin, 1997.

PHILLIPS, P.C.B. \& PERRON, P. Testing for unit root in time series regression. Biometrica, 75, p.335-346, 1988.

SAPRA, Haresh. Hedge Disclosures, Futures Prices and Production Distortions. Working Paper, Graduate School of Business University of Chicago, 2002.
SECURITIES AND EXCHANGE COMISSION (SEC). 1997. Disclosure of Accounting Policies for Derivatives Financial Instruments and Derivative Commodity Instruments and Disclosure of Quantitative and Qualitative Information about Market Risk Inherent in Derivative Financial Instruments, Other Financial Instruments, and Derivative Commodity Instruments. Releases No 33-7386; 34-38223; IC-22487; FR-48; International Series No 1047; File No S7-35-95 (January 31, 1997). Washington, D.C.: Securities and Exchange Comission.

SECURITIES AND EXCHANGE COMISSION (SEC). 1995. Proposed Amendments to Require Disclosures of Accounting Policies for Derivative Financial Instruments and Derivative Commodity Instruments and Disclosure of Qualitative and Quantitative Information about Market Risk in Derivative Financial Instruments, Other Financial Instruments, and Derivative Commodity Instruments. Release No 7250; December 28, 1995 Washington, D.C.: Securities and Exchange Comission; 61 FR 578, January 8, 1996.

STRAUSS, Mel. Examining FAS 133. US Bankers Magazine. Setembro de 1998. Nova York.

WILSON, Arlette C. The Decision on Derivatives. Journal of Accountancy. Novembro de 1998. AICPA, New Jersey. 